\title{
Patient-Reported Outcomes Through 1 Year of an HIV-1 Clinical Trial Evaluating Long-Acting Cabotegravir and Rilpivirine Administered Every 4 or 8 Weeks (ATLAS-2M)
}

\author{
Vasiliki Chounta ${ }^{1}$ - Edgar T. Overton ${ }^{2} \cdot$ Anthony Mills $^{3} \cdot$ Susan Swindells ${ }^{4} \cdot$ Paul D. Benn $^{1} \cdot$ Simon Vanveggel $^{5}$. \\ Rodica van Solingen-Ristea ${ }^{5}$ Y Yuanyuan Wang ${ }^{6} \cdot$ Krischan J. Hudson $^{7,8} \cdot$ Mark S. Shaefer $^{7,9} \cdot$ David A. Margolis $^{7,10}$. \\ Kimberly Y. Smith ${ }^{7}$. William R. Spreen ${ }^{7}$
}

Accepted: 28 April 2021 / Published online: 31 May 2021

(c) The Author(s) 2021

\begin{abstract}
Background Advances in HIV-1 therapeutics have led to the development of a range of daily oral treatment regimens, which share similar high efficacy rates. Consequently, more emphasis is being placed upon the individual's experience of treatment and impact on quality of life. The first long-acting injectable antiretroviral therapy for HIV-1 (long-acting cabotegravir + rilpivirine $[\mathrm{CAB}+\mathrm{RPV} \mathrm{LA}]$ ) may address challenges associated with oral treatment for HIV-1, such as stigma, pill burden/fatigue, drug-food interactions, and adherence. Patient-reported outcomes (PROs) collected in an HIV-1 clinical trial (ATLAS-2M; NCT03299049) comparing participants' experience with two dosing regimens (every 4 weeks [Q4W] vs. every 8 weeks [Q8W]) of CAB + RPV LA are presented herein.

Methods PRO endpoints evaluated through 48 weeks of therapy included treatment satisfaction (HIV Treatment Satisfaction Questionnaire [HIVTSQ]), treatment acceptance ("General Acceptance" domain of the Chronic Treatment Acceptance [ACCEPT ${ }^{\circledR}$ ] questionnaire), acceptability of injections (Perception of Injection [PIN] questionnaire), treatment preference (questionnaire), and reasons for switching to/continuing long-acting therapy (exploratory endpoint; questionnaire). Participants were randomized 1:1 to receive CAB + RPV LA Q8W or Q4W. Results were stratified by prior CAB + RPV exposure in either preplanned or post hoc analyses.

Results Overall, 1045 participants were randomized to the Q8W $(n=522)$ and Q4W $(n=523)$ regimens; 37\% $(n=391 / 1045)$ had previously received $\mathrm{CAB}+\mathrm{RPV}$ in ATLAS. For participants without prior CAB + RPV exposure, large increases from baseline were reported in treatment satisfaction in both long-acting arms (HIVTSQ status version), with Q8W dosing statistically significantly favored at Weeks $24(p=0.036)$ and $48(p=0.004)$. Additionally, improvements from baseline were also observed in the "General Acceptance" domain of the ACCEPT questionnaire in both long-acting arms for participants without prior $\mathrm{CAB}+\mathrm{RPV}$ exposure; however, no statistically significant difference was observed between arms at either timepoint (Week 24, $p=0.379$; Week 48, $p=0.525)$. Significant improvements $(p<0.001)$ in the "Acceptance of Injection Site Reactions" domain of the PIN questionnaire were observed from Week 8 to Weeks 24 and 48 in both arms for participants without prior $\mathrm{CAB}+\mathrm{RPV}$ exposure. Participants with prior $\mathrm{CAB}+\mathrm{RPV}$ exposure reported high treatment satisfaction (mean [HIVTSQ status version]: Q8W 62.2/66.0; Q4W 62.0/66.0), treatment acceptance (mean: Q8W 89.3/100; Q4W 91.2/100), and acceptance of injection site reactions (mean [5 = not at all acceptable; $1=$ totally acceptable]: Q8W 1.72; Q4W 1.59) at baseline/Week 8 that were maintained over time. Participants without prior CAB + RPV exposure who received Q8W dosing preferred this regimen over oral CAB + RPV $(98 \%, n=300 / 306)$. Among those with prior Q4W exposure, 94\% $(n=179 / 191)$ preferred Q8W dosing versus Q4W dosing $(3 \%, n=6 / 191)$ or oral CAB + RPV $(2 \%, n=4 / 191)$.

Conclusions Both long-acting regimens provided high treatment satisfaction and acceptance, irrespective of prior $\mathrm{CAB}+\mathrm{RPV}$ exposure, with most participants preferring Q8W dosing over both the Q4W regimen and their previous daily oral regimen. The PRO data collected at Week 48 support the therapeutic potential of CAB + RPV LA.
\end{abstract}

Funding ViiV Healthcare and Janssen.

Trial Registration ATLAS-2M: ClinicalTrials.gov NCT03299049, registered October 2, 2017.

Extended author information available on the last page of the article 


\section{Plain Language Summary}

Developments in HIV-1 treatment have resulted in effective daily oral medications. However, life-long pill taking can come with several challenges. These include having a daily reminder of living with HIV-1. Treatment satisfaction is important to consider when evaluating a new medicine. This is because it can affect people's quality of life. The purpose of this study was to evaluate people's experiences with the first long-acting injectable medicine for HIV-1. The medicine is called cabotegravir + rilpivirine long-acting (CAB + RPV LA). Over approximately 1 year, this study measured people's satisfaction and experiences while receiving injections of CAB +RPV LA. Injections were given either every 4 weeks or every 8 weeks. The study included people who had never had CAB + RPV LA, as well as people who were already receiving CAB $+\mathrm{RPV}$ LA. For people new to CAB + RPV LA, their satisfaction increased compared with their previous medication. They also had improvements in their experiences of injection site reactions throughout the study. For people who were already receiving $\mathrm{CAB}+\mathrm{RPV}$ LA, their high satisfaction with this treatment and tolerability of injection site reactions were maintained over time. Overall, improvements were similar between people receiving injections every 4 weeks and people receiving injections every 8 weeks. People with experience of both injection schedules tended to prefer to receive injections every 8 weeks. These results show that CAB + RPV LA can provide quality-of-life improvements for people who have HIV-1.

\section{Key Points for Decision Makers}

Participants with no previous experience with cabotegravir + rilpivirine $(\mathrm{CAB}+\mathrm{RPV})$ reported increases in treatment satisfaction for both the every 8 weeks regimen and every 4 weeks regimen over their previous daily oral regimen.

Participants were found to be satisfied with $\mathrm{CAB}+\mathrm{RPV}$ long-acting (LA) as a treatment for the maintenance of virologic suppression across a range of patient-reported outcomes.

For participants with exposure to both dosing regimens of CAB + RPV LA, most participants preferred the every 8 weeks regimen over the every 4 weeks regimen.

\section{Introduction}

Advances in the efficacy of antiretroviral therapy [1] have led to a shift in treatment focus to emphasize convenience, tolerability, and overall acceptability of HIV therapy [2]. This has culminated in the development of efficacious single-tablet daily oral regimens, which offer simplicity and convenience [3]. Despite this, the need for life-long daily pill-taking can present substantial challenges that can be broadly grouped into three categories: adherence issues [4]; emotional issues, including stigmatization concerns and the daily reminder of HIV status [5]; and medical issues, such as difficulty swallowing, drug-food and drug-drug interactions, and pill burden [6]. These challenges can impact the likelihood of treatment failure, with poor adherence to antiretroviral therapy linked to the emergence of resistance and virologic failure [7-10]. Consequently, there is considerable interest across a range of patient demographics in long-acting therapies, in particular, injectable long-acting therapies, which may help to alleviate some of the challenges associated with daily oral therapy [11-15].

Cabotegravir (CAB), an HIV integrase strand transfer inhibitor, and rilpivirine (RPV), a non-nucleoside reverse transcriptase inhibitor, are the first intramuscular long-acting antiretroviral regimen ( $\mathrm{CAB}+\mathrm{RPV} \mathrm{LA})$ for the maintenance of virologic suppression in people living with HIV-1 and can be dosed monthly or every 2 months [16-18]. Currently, CAB + RPV LA is approved for use in Australia, the USA, Canada, and the European Union for adults with HIV-1. Both CAB and RPV are also available as separate oncedaily oral tablets, intended for use as an oral lead-in to assess safety and tolerability prior to initiation of the long-acting regimen. These tablets can also be used as a bridge for the long-acting regimen if there are planned interruptions to treatment [16-18].

The phase 2b LATTE-2 study (NCT02120352) demonstrated that CAB + RPV LA dosed every 4 weeks $(\mathrm{Q} 4 \mathrm{~W})$ or every 8 weeks $(\mathrm{Q} 8 \mathrm{~W})$ was as effective as continuing oral $\mathrm{CAB}+$ abacavir/lamivudine for maintaining virologic suppression [19]. Qualitative interviews with 39 participants revealed that the long-acting regimen was perceived as a highly acceptable and desirable alternative to oral therapy, with convenience and peace of mind reported as the primary contributing factors [20]. CAB + RPV LA has also been evaluated in three phase 3 studies. ATLAS (NCT02951052) and FLAIR (NCT02938520), both individually $[16,17]$ and in a pooled analysis [21], demonstrated the noninferiority of CAB + RPV LA Q4W for the maintenance of virologic suppression versus continuing daily oral therapy. Moreover, a pooled analysis of patient-reported outcomes (PROs) from these two studies found high levels of participant satisfaction, acceptance, and tolerability with $\mathrm{CAB}+\mathrm{RPV}$ LA, with most participants preferring the long-acting regimen to oral therapy [22]. Subsequently, the ATLAS-2M (NCT03299049) study demonstrated the noninferiority of $\mathrm{CAB}+\mathrm{RPV}$ LA Q8W versus Q4W dosing for the maintenance of virologic suppression [18]. 
Increased patient satisfaction has been shown to improve adherence [23], which contributes to the maintenance of long-term HIV suppression as well as preventing drug resistance, slowing disease progression, and reducing mortality $[9,10,24-26]$. PROs can aid treatment optimization as they provide key insights into quality-of-life issues that have the potential to influence adherence to life-long treatment. Previous clinical trials have demonstrated that participants switching between contemporary oral antiretroviral regimens experience modest improvements in PROs [27-29]. Here, we evaluate and compare participants' experience with the two dosing regimens of CAB + RPV LA (Q4W vs. Q8W) using PRO data from preplanned and post hoc analyses in ATLAS-2M.

\section{Methods}

\subsection{Study Design and Participants}

ATLAS-2M (electronic supplementary material Figure S1) is an ongoing, multicenter, phase $3 b$, randomized, open-label study investigating whether CAB + RPV LA Q8W is noninferior to CAB + RPV LA Q4W. The eligibility criteria, study design, and methodology for participant recruitment have been reported previously in the primary clinical manuscript [18]. Briefly, eligible participants were adults ( $\geq 18$ years) with antiretroviral therapy experience, virologic suppression (HIV-1 RNA $<50$ copies $/ \mathrm{mL}$ ), and no history of resistance. Participants from either the standard of care or CAB + RPV LA (Q4W) arms of the ATLAS study (NCT02951052) were also eligible to enter ATLAS-2M, provided they had received treatment through a minimum of 52 weeks in the ATLAS study and were virologically suppressed at ATLAS$2 \mathrm{M}$ screening.

After screening, participants were randomized 1:1 to receive $\mathrm{CAB}+\mathrm{RPV} \mathrm{LA}$ in the maintenance phase, dosed either Q8W (CAB $600 \mathrm{mg}+\mathrm{RPV} 900 \mathrm{mg}$ ) or Q4W (CAB $400 \mathrm{mg}+$ RPV $600 \mathrm{mg}$ ). Randomization was stratified by three categories of prior CAB + RPV (oral + intramuscular) exposure $(0,1-24$, and $>24$ weeks) to account for those individuals entering from the ATLAS study and the difference in treatments assessed at baseline (participants transitioning from the randomized Q4W arm in ATLAS had > 24 weeks of exposure to $\mathrm{CAB}+\mathrm{RPV}$, whereas all but one participant transitioning from the randomized standard of care arm had $<24$ weeks of exposure to CAB + RPV whilst waiting for enrollment into ATLAS-2M [one participant had 24-48 weeks of exposure]). ATLAS-2M was conducted in accordance with the principles founded in the Declaration of Helsinki [30] and with Good Clinical Practice [31]. All participants provided written informed consent, and the protocol was approved by an institutional review board or ethics committee.

\subsection{Assessments and Endpoints}

Several PRO instruments (Table 1) were included at prespecified timepoints in the ATLAS-2M study to assess tolerability and acceptability of injections, treatment satisfaction, acceptance, and preference. The PRO instruments utilized are detailed below alongside references to studies validating their psychometric properties.

\subsubsection{Perception of Injection (PIN) Questionnaire}

The acceptability and tolerability of injections and injection site reactions (ISRs) was assessed using the Perception of Injection (PIN) questionnaire. The PIN questionnaire was modified for gluteal intramuscular injection from the Vaccinees' Perception of Injection (VAPI) questionnaire, developed and validated to assess perception and acceptance of influenza vaccination [32]. A validation publication assessing its use in an HIV population is under preparation. The questionnaire comprises 21 items across four dimensions taken from the VAPI, "Acceptance of ISRs," "Bother of ISRs," "Sleep," and "Leg movement," and five new individually reported items related to willingness to receive an HIV injectable treatment at the following visit, pain during injection, satisfaction with the mode of treatment administration, and anxiety before and after the injection. Answers to the questionnaire are reported as scores ranging from 1 to 5 , representing, in order, "totally acceptable," "very acceptable," "moderately acceptable," "a little acceptable," and "not at all acceptable." The mean of all items within a dimension was taken to give the score of the dimension. Only the "Acceptance of ISRs" domain was included in this analysis and remained unchanged from the original version.

\subsubsection{Chronic Treatment Acceptance Questionnaire (ACCEPT ${ }^{\oplus}$ )}

How participants weigh the advantages and disadvantages of long-term medications was assessed using the "General Acceptance" domain of the Chronic Treatment Acceptance (ACCEPT) questionnaire, validated in populations receiving treatment for a chronic disease (including HIV-1) [33, 34]. The domain consists of the following three items:

1. 'Do you agree with the following statement: "My medication has more advantages than disadvantages"?'

2. "Given the advantages and disadvantages of your medication, do you consider it to be an acceptable solution?" 
Table 1 ATLAS-2M PRO instruments

\begin{tabular}{|c|c|c|c|c|}
\hline PRO instrument & Assessment & Timepoints measured & $\begin{array}{l}\text { Stratified by prior } \\
\text { CAB + RPV exposure } \\
\text { (prespecified analysis) }\end{array}$ & Endpoint \\
\hline PIN questionnaire & Perception of pain and ISRs & $\begin{array}{l}\text { Week } 8 \text {, Week } 24 \text {, Week } 48 \text { (or } \\
\text { withdrawal) }\end{array}$ & $\mathrm{No}^{\mathrm{a}}$ & Secondary \\
\hline ACCEPT & Patient acceptance of treatment & $\begin{array}{l}\text { Baseline, Week 24, Week } 48 \text { (or } \\
\text { withdrawal) }\end{array}$ & Yes & Secondary \\
\hline HIVTSQs & $\begin{array}{l}\text { Patient satisfaction with HIV } \\
\text { treatment }\end{array}$ & $\begin{array}{l}\text { Baseline, Week 24, Week } 48 \text { (or } \\
\text { withdrawal) }\end{array}$ & Yes & Secondary \\
\hline HIVTSQc & $\begin{array}{l}\text { Change in patient satisfaction with } \\
\text { HIV treatment from previous } \\
\text { regimen }\end{array}$ & Week 48 (or withdrawal) & $\mathrm{No}^{\mathrm{a}, \mathrm{b}}$ & Secondary \\
\hline Preference for HIV Treatment & $\begin{array}{l}\text { Patient preference for } \mathrm{CAB}+\mathrm{RPV} \\
\mathrm{LA} \text { vs. oral } \mathrm{CAB}+\mathrm{RPV} \text { and } \\
\text { reasons for preference }\end{array}$ & Week 48 (or withdrawal) & Yes & Secondary \\
\hline HAT-QoL ${ }^{c}$ & $\begin{array}{l}\text { Patient life satisfaction, disclosure } \\
\text { worries, and HIV medication }\end{array}$ & $\begin{array}{l}\text { Baseline, Week 24, Week } 48 \text { (or } \\
\text { withdrawal) }\end{array}$ & Yes & Secondary \\
\hline $\begin{array}{l}\text { Reason for switch/continua- } \\
\text { tion (single question) }\end{array}$ & $\begin{array}{l}\text { Patient reasoning for switching to } \\
\text { LA therapy from oral therapy/ } \\
\text { continuing LA therapy }\end{array}$ & Baseline & $\mathrm{No}^{\mathrm{b}}$ & Exploratory \\
\hline
\end{tabular}

ACCEPT Chronic Treatment Acceptance Questionnaire, CAB cabotegravir, ISR injection site reaction, HAT-QoL HIV/AIDS-targeted quality of life, HIVTSQs/c HIV Treatment Satisfaction Questionnaire (status version)/(change version), LA long-acting, PIN Perception of Injection, PRO patient-reported outcome, $Q 4 W$ every 4 weeks, $R P V$ rilpivirine, $S o C$ standard of care

${ }^{a}$ Analyses stratified by prior CAB + RPV exposure were post hoc

${ }^{b}$ Prespecified analysis was stratified by prior regimen (randomized CAB + RPV LA Q4W in ATLAS vs. SoC [randomized to SoC in ATLAS/ receiving $\mathrm{SoC}$ in routine clinic])

${ }^{c}$ Data not shown. The results remained generally stable across all visits, irrespective of prior $\mathrm{CAB}+\mathrm{RPV}$ exposure, with no significant changes from baseline after adjusting for prespecified covariates

3. "Are you convinced that in the long term, it is worth taking your medications?"

Participant responses are rated on a 5-point Likert scale, with 5 representing "I don't know" and scores of 1 through 4 representing increments in agreement or acceptance from "totally disagree/not at all acceptable/not at all convinced" through to "totally agree/totally acceptable/totally convinced." An aggregate score was then produced from the individual item scores and linearly transformed to range from 0 to 100 , with higher scores representing greater agreement or acceptance.

\subsubsection{HIV Treatment Satisfaction Questionnaire (Status and Change Versions)}

Treatment satisfaction was assessed using a 12 -item questionnaire adapted from the original 10-item HIV Treatment Satisfaction Questionnaire (HIVTSQ), which was validated in a population with HIV-1 [35]. The 12-item adaptation was previously used in the ATLAS [17] and FLAIR [16] studies and validated in the LATTE-2 study [19] (publication pending). The two additional items were added to specifically address the mode of administration of CAB + RPV LA (i.e., injectable intramuscular dosing). These were:

1. "How easy or difficult have you been finding your treatment to be recently?"

2. "How satisfied are you with the amount of discomfort or pain involved with your present form of treatment?"

HIVTSQ status version (HIVTSQs) instructs participants to rank their answers on a 6-point Likert scale (ranging from 6 ["very satisfied"] to 0 ["very dissatisfied"]). In contrast, HIVTSQ change version (HIVTSQc) asks participants to compare the HIV therapy they were receiving prior to entering ATLAS-2M with their current treatment and rank their answers from 3 ("much more satisfied now") to -3 ("much less satisfied now"). HIVTSQc can help to account for the potentially high baseline values recorded with HIVTSQs and prevent measurement limitations (ceiling effects), which can be associated with satisfaction measurements and mask the magnitude of treatment effects (maximum or near-maximum satisfaction at baseline resulting in recording little or no improvement at later timepoints) [36, 37]. In both versions, item scores were added together to give a total aggregate summary score (HIVTSQs: 0 [minimum, very dissatisfied], 
66 [maximum, very satisfied]; HIVTSQc: - 33 [minimum, much less satisfied now], 33 [maximum, much more satisfied now]).

\subsubsection{Preference}

To assess participant preference, a single-item question evaluating treatment preference, along with questions evaluating attributes supporting this preference, was included. The question read:

1. "Based on your experience, which HIV treatment do you prefer?"

The single-item question had multiple closed-format response options. For participants without prior $\mathrm{CAB}+\mathrm{RPV}$ exposure, response options were between the $\mathrm{Q} 8 \mathrm{~W}$ or $\mathrm{Q} 4 \mathrm{~W}$ regimen they received in ATLAS-2M and their previous daily oral regimen. For those with prior $\mathrm{CAB}+\mathrm{RPV}$ exposure in the Q8W arm, response options were between the Q8W regimen, the Q4W regimen (received previously in ATLAS), and their previous daily oral regimen. For those with prior exposure in the Q4W arm, only Q4W dosing and daily oral dosing were response options.

\subsubsection{Reason for Switch}

To explore the reasons why participants elected to switch to or continue long-acting treatment, a single-item question was administered. The question read:

1. "You have chosen to participate in a clinical study where your daily oral HIV medication will be switched to a long-acting injectable HIV medication. What are the main reasons for your willingness to switch to a longacting injectable HIV medication from your current oral HIV medication?"

Or

2. "You have chosen to participate in a clinical study where you will continue to receive long-acting injectable HIV medication. What are the main reasons for your willingness to continue with a long-acting injectable HIV medication?"

The multiple closed-format response options are shown in electronic supplementary material Figure S2.

\subsection{Statistical Analysis}

Descriptive statistics were used to summarize questionnaire scores for each visit (baseline, Week 8, Week 24, and Week 48). The prespecified analysis of ACCEPT and HIVTSQs was stratified by prior CAB + RPV exposure ( 0 weeks vs. $\geq 1$ week of exposure) to account for the different treatments assessed at baseline. A post hoc analysis of PIN and HIVTSQc was also stratified by prior $\mathrm{CAB}+\mathrm{RPV}$ exposure ( 0 weeks vs. $\geq 1$ week of exposure). An analysis of covariance (ANCOVA) model was used to examine treatment differences at each visit for HIVTSQs, ACCEPT, and PIN, and was adjusted for select covariates. For HIVTSQs and ACCEPT, these included baseline score, sex at birth (female, male), age $(<50, \geq 50$ years), and race (white, non-white). For the post hoc analysis of PIN, treatment differences were adjusted for Week 8 score, sex at birth (female, male), age $(<50, \geq 50$ years), and race (white, non-white). An ANOVA model was used to assess treatment differences at Week 48 for HIVTSQc and was adjusted for sex at birth (female, male), age ( $<50, \geq 50$ years), and race (white, non-white). These models were also adjusted for prior exposure to CAB + RPV (1-24, > 24 weeks) when examining nonnaive participants to $C A B+R P V$. The prespecified analysis of treatment differences for PIN was adjusted for Week 8 score, sex at birth (female, male), age $(<50, \geq 50$ years), race (white, non-white), and prior exposure to $C A B+R P V$ $(0,1-24,>24$ weeks). $P$ values and $95 \%$ confidence intervals (CIs) for the treatment differences between groups are reported. Change in PIN acceptance score from Week 8 to Week 24 and Week 48 were evaluated for each treatment group using a Wilcoxon signed-rank test. Missing data were imputed using a last-observation-carried-forward approach including measures assessed at time of withdrawal. For the preference questionnaire, the prespecified analysis was the respondent's analysis (participants that responded at Week 48 or withdrawal). For the exploratory endpoint (reason for switch), proportions are reported for observed cases without imputation, statistical modeling, or testing. A statistical appendix can be found in the electronic supplementary material.

\section{Results}

\subsection{Baseline Characteristics}

The intention-to-treat exposed population consisted of 1045 participants (Q8W, $n=522$; Q4W, $n=523$ ). Baseline characteristics have been reported previously and were similar between dosing arms [18]. Overall, 37\% $(n=391 / 1045)$ of participants entered ATLAS-2M with prior CAB + RPV experience in ATLAS, most of whom $(65 \%, n=253 / 391)$ had $>48$ weeks of prior exposure. 


\subsection{Acceptability of ISRs (PIN Questionnaire)}

At Week 8, most participants reported their acceptance of pain (Q8W 67\%; Q4W 69\%) and local reactions (Q8W 81\%; Q4W 82\%) as either "totally acceptable" or "very acceptable" ("Acceptance of ISRs" domain of the PIN questionnaire) (Fig. 1a, b). Across both long-acting groups, a statistically significant improvement $(p<0.005)$ in the acceptability of ISRs was observed from Week 8 to Weeks 24 and 48 (Fig. 2a), with $77 \%$ of participants in either treatment arm (Q8W, $n=399 / 515$; Q4W, $n=398 / 515)$ rating pain as "totally acceptable" or "very acceptable" at Week 48 (Fig. 1b). Further, 85\% ( $n=439 / 515)$ and 84\% $(n=434 / 515)$ of participants rated their local reactions as "totally acceptable" or "very acceptable" at Week 48 in the Q8W and Q4W arms, respectively (Fig. 1a). No statistically significant difference in adjusted mean change from Week 8 to Weeks 24 and 48 in acceptability of ISRs was observed between the long-acting groups (Week 24 adjusted difference [Q8W - Q4W] [95\% CI] 0.01 [ 0.07 to 0.10], $p=0.768$; Week 48 adjusted difference [Q8W-Q4W] [95\% CI] -0.04 [ -0.13 to 0.05$], p=0.391)$.

When stratified by prior CAB + RPV exposure, statistically significant improvements $(p<0.001)$ from Week 8 to Weeks 24 and 48 in the acceptability of ISRs were observed for participants with no prior exposure across both longacting groups (Fig. 2b). For participants with prior exposure, representing participants transitioning from the ATLAS study, mean (standard deviation [SD]) acceptability of ISRs was high at Week 8 (Q8W 1.72 [0.842]; Q4W 1.59 [0.741]) and was maintained through Weeks 24 (Q8W 1.76 [0.805]; Q4W 1.67 [0.740]) and 48 (Q8W 1.68 [0.840]; Q4W 1.65 [0.779]) in both long-acting groups.
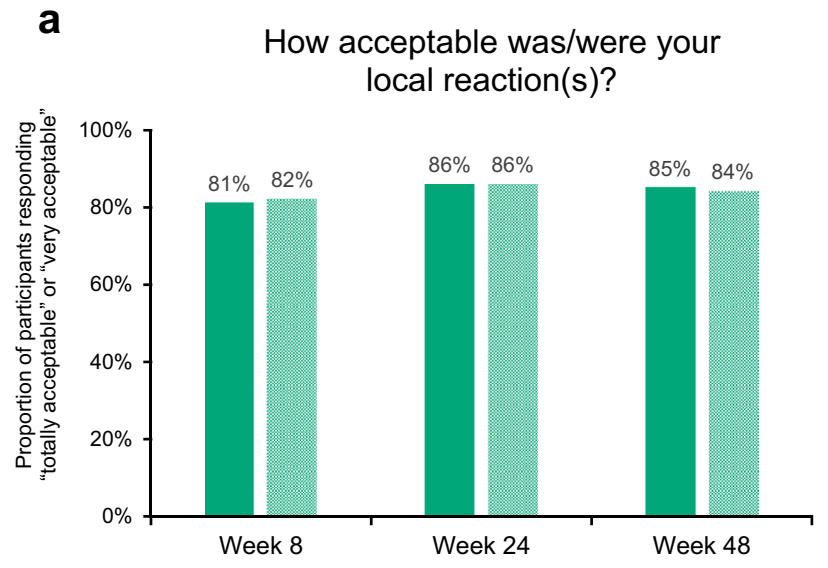

CAB+RPV LA Q8W

\subsection{Treatment Acceptance ("General Acceptance" Domain of ACCEPT)}

For participants without prior exposure to $\mathrm{CAB}+\mathrm{RPV}$, baseline scores in the "General Acceptance" domain of the ACCEPT questionnaire, evaluating participants' acceptance of prior daily oral therapy, were similar between both long-acting groups (mean [SD]: Q8W 81.5/100 [25.23]; Q4W 81.8/100 [25.98]). At Week 24 and Week 48, marked improvements from baseline in treatment acceptance were observed across both long-acting groups (adjusted mean change from baseline [95\% CI] [percentage change from baseline, calculated as the adjusted mean change from baseline divided by the mean baseline score multiplied by 100]: Q8W: Week 24, 5.8 [3.2-8.5] [+7\%]; Week 48, 6.8 [4.3-9.3] [+ 8\%]; Q4W: Week 24, 4.2 [1.5-6.8] [+5\%]; Week 48, 5.7 [3.2-8.1] [+7\%]) (Fig. 3a). As per the preplanned statistical analysis, no significant differences were observed between the two long-acting groups in change from baseline in treatment acceptance at Weeks $24(p=0.379)$ and $48(p=0.525)$.

For participants with prior $\mathrm{CAB}+\mathrm{RPV}$ exposure, baseline "General Acceptance" scores, evaluating participants' acceptance of prior Q4W long-acting therapy, were high and similar across long-acting groups (mean baseline [SD]: Q8W 89.3/100 [20.03]; Q4W 91.2/100 [16.74]). Scores remained high at Weeks 24 and 48 without significant differences between treatment groups (Fig. 3b). For most of these participants $(65 \%, n=253 / 391)$, this represented $\sim 96$ weeks of cumulative experience of long-acting therapy across ATLAS and ATLAS-2M.

\section{b}

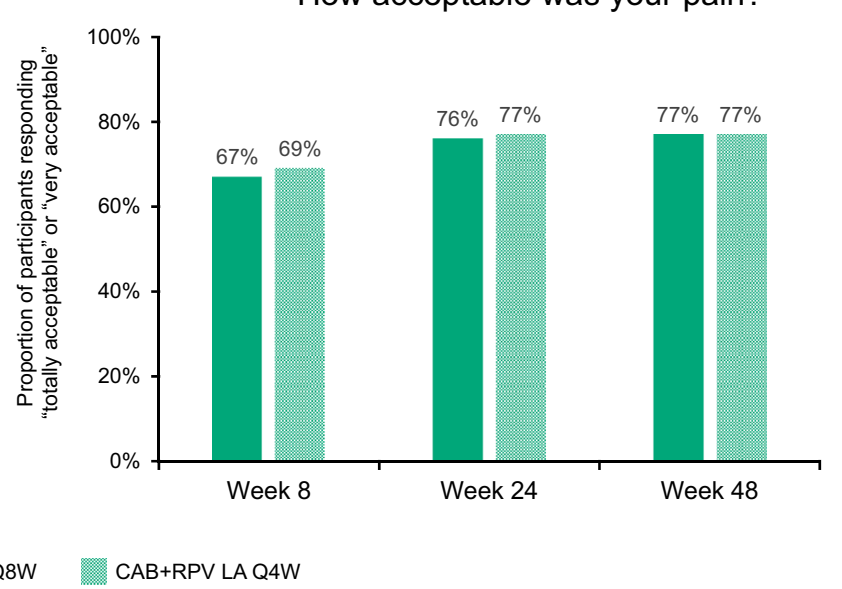

Fig. 1 "Acceptance of ISRs" domain of the PIN questionnaire through Week $48^{\mathrm{a}}$. $C A B$ cabotegravir, ISR injection site reaction, $L A$ long-acting, PIN Perception of Injection, $Q 4 W$ every 4 weeks,
$Q 8 W$ every 8 weeks, $R P V$ rilpivirine. ${ }^{a}$ Week 8 : Q8W, $n=514$; Q4W, $n=515$; Week 24: Q8W, $n=515$; Q4W, $n=515$; Week 48: Q8W, $n=515 ; \mathrm{Q} 4 \mathrm{~W}, n=515$ 
a

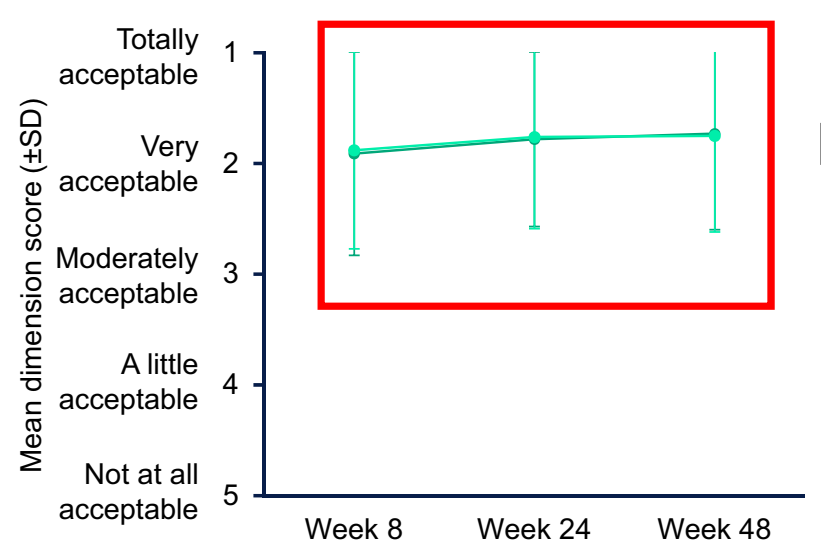

b

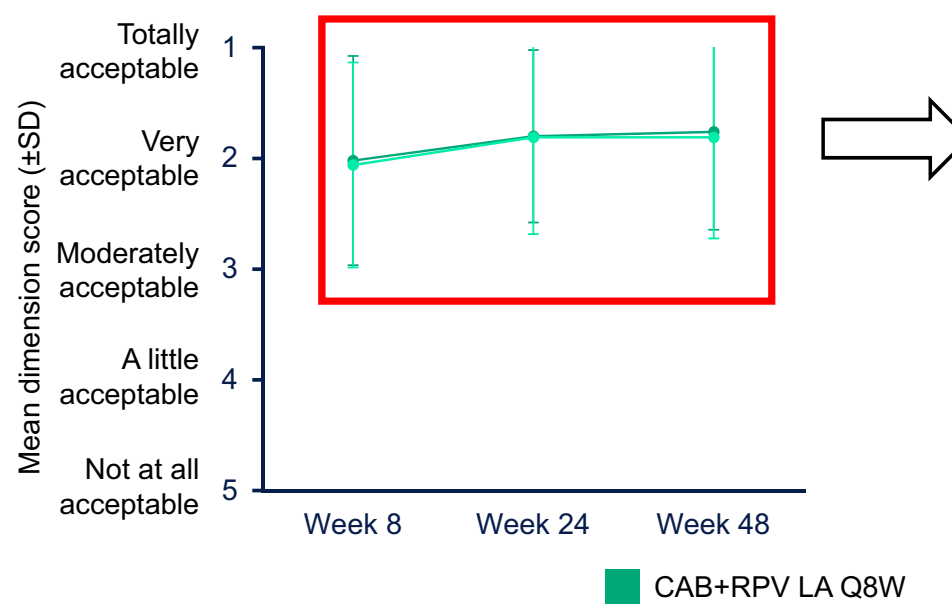

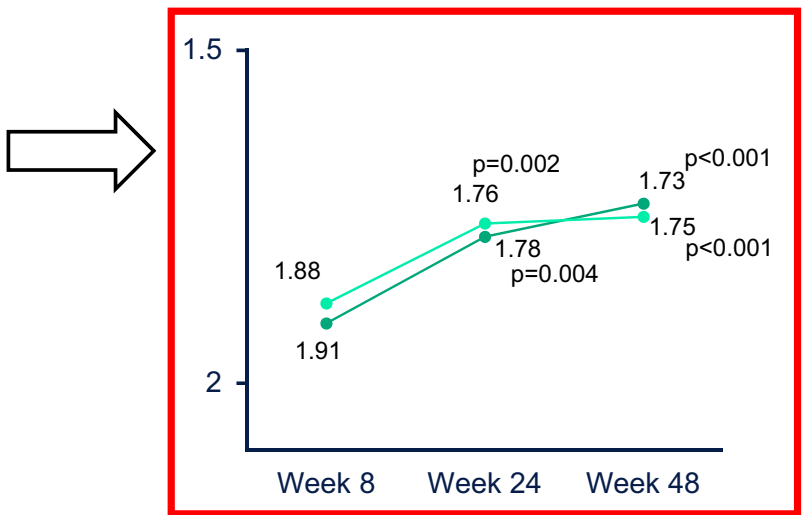

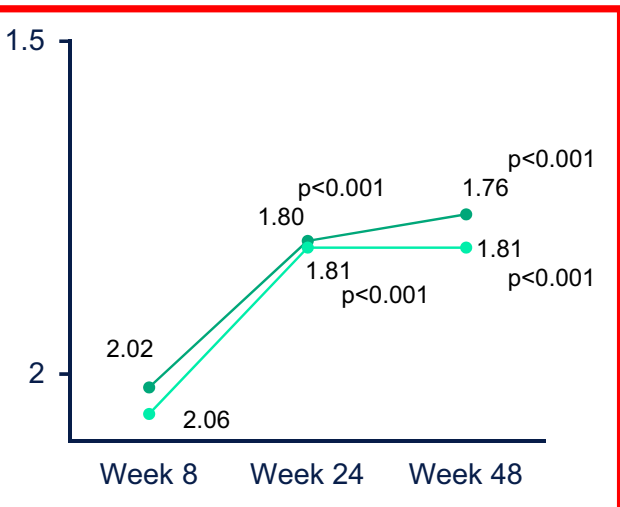

CAB+RPV LA Q4W
Fig. 2 Mean "Acceptance of ISRs" domain of the PIN questionnaire score through Week 48 for the total population ${ }^{\mathrm{a}}$ (a) and participants with no prior $\mathrm{CAB}+\mathrm{RPV}$ exposure ${ }^{\mathrm{b}}$ (b). $C A B$ cabotegravir, ISR injection site reaction, $L A$ long-acting, PIN Perception of Injection, $Q 4 W$ every 4 weeks, $Q 8 W$ every 8 weeks, $R P V$ rilpivirine, $S D$ standard deviation. ${ }^{a}$ Week 8: Q8W, $n=514$; Q4W, $n=515$; Week 24: Q8W, $n=515$; Q4W, $n=515$; Week 48: Q8W, $n=515$; Q4W, $n=515$. $P$ values correspond to the Wilcoxon signed-rank test used to com-

\subsection{Treatment Satisfaction (HIVTSQs/c)}

For participants without prior $\mathrm{CAB}+\mathrm{RPV}$ exposure, baseline HIVTSQs mean (SD) scores were similar between treatment groups (Q8W 57.73/66.00 [9.21]; Q4W 56.72/66.00 [9.34]). At both Week 24 and Week 48, HIVTSQs total scores were markedly improved from baseline (adjusted mean change from baseline $[95 \% \mathrm{CI}$ ] [percentage change from baseline, calculated as the adjusted mean change from baseline divided by the mean baseline score multiplied by 100]: Q8W: Week 24, 5.07 [4.36-5.78] [+9\%]; Week 48, 4.86 [4.02-5.69] [+8\%]; Q4W: Week 24, 4.00 [3.29-4.70] [+7\%]; Week 48, 3.12 [2.29-3.95] [+6\%]) (Fig. 4a), with statistically significant differences in the adjusted mean change from baseline favoring Q8W dosing at both timepoints (Week 24, $p=0.036$; Week 48, $p=0.004$ ). At Week pare the Week 24 and Week 48 scores with the Week 8 scores. $P$ values are derived for "acceptance" only and not adjusted for multiple testing. ${ }^{b}$ Week 8: Q8W, $n=320$; Q4W, $n=321$; Week 24: Q8W, $n=321$; Q4W, $n=321$; Week 48: Q8W, $n=321$; Q4W, $n=321$. $P$ values correspond to the Wilcoxon signed-rank test used to compare the Week 24 and Week 48 scores with the Week 8 scores. $P$ values are derived for "acceptance" only and not adjusted for multiple testing

48 , treatment satisfaction had improved from baseline in nine of the 12 individual items, with Q8W dosing scoring equal to or higher than the Q4W dosing arm in all nine items (electronic supplementary material Figure S3).

For participants with prior CAB + RPV exposure, HIVTSQs mean (SD) scores were high at baseline (Q8W 62.22/66.00 [5.41]; Q4W 61.98/66.00 [6.72]) and remained stable from Week 24 to Week 48 (Fig. 4b). By Week 48 of ATLAS-2M, most of these participants $(65 \%, n=253 / 391)$ who had transitioned from the ATLAS study had 96 weeks of cumulative experience of long-acting therapy. No significant differences $(p>0.05)$ in HIVTSQs were found between the long-acting groups at any timepoint. At Week 48, HIVTSQc scores, used to account for ceiling effects [37], showed an improvement in treatment satisfaction compared with the pre-randomization treatment for both groups, 
a

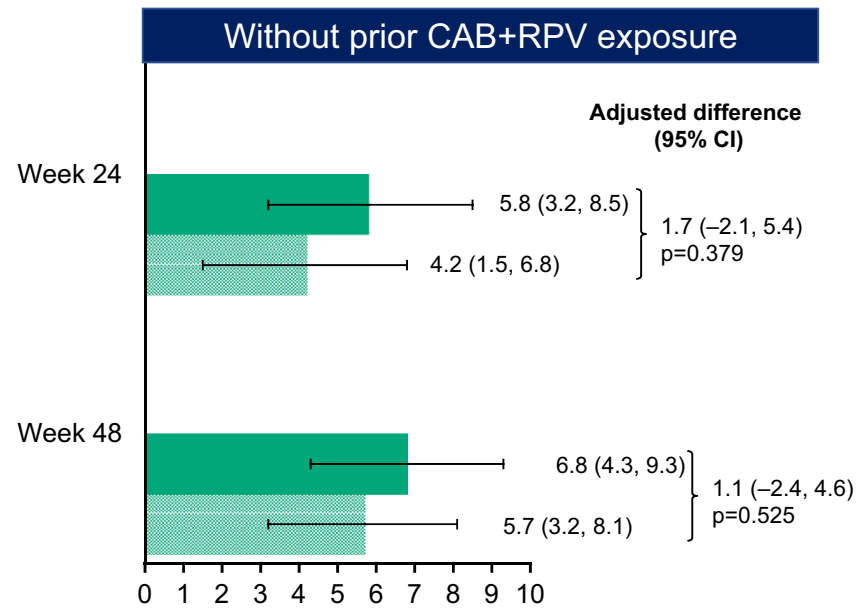

b

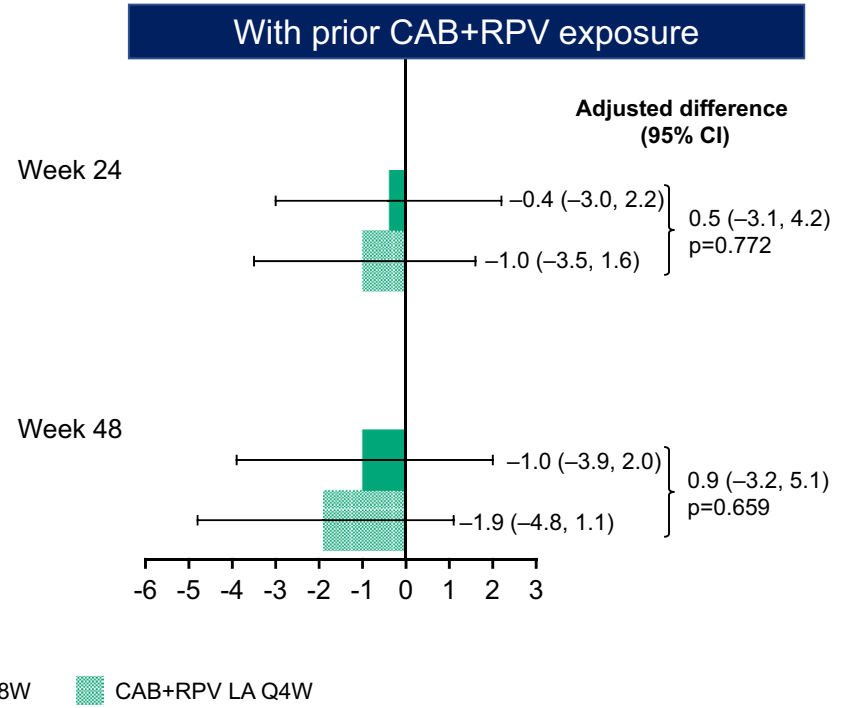

Fig. 3 Adjusted mean change from baseline in "General Acceptance" score of the ACCEPT questionnaire by visit for participants without prior $\mathrm{CAB}+\mathrm{RPV}$ exposure (a) and with prior $\mathrm{CAB}+\mathrm{RPV}$ exposure $(\mathbf{b})^{\mathrm{a}}$. ACCEPT Chronic Treatment Acceptance Questionnaire, $A N C O V A$ analysis of covariance, $C A B$ cabotegravir, $C I$ confidence interval, $L A$ long-acting, $Q 4 W$ every 4 weeks, $Q 8 W$ every 8 weeks, $R P V$ rilpivirine. ${ }^{a}$ No prior exposure, Week 24: Q8W, $n=319$; Q4W, $n=323$; Week 48: Q8W, $n=319$; Q4W, $n=324$; prior exposure,

\section{a}

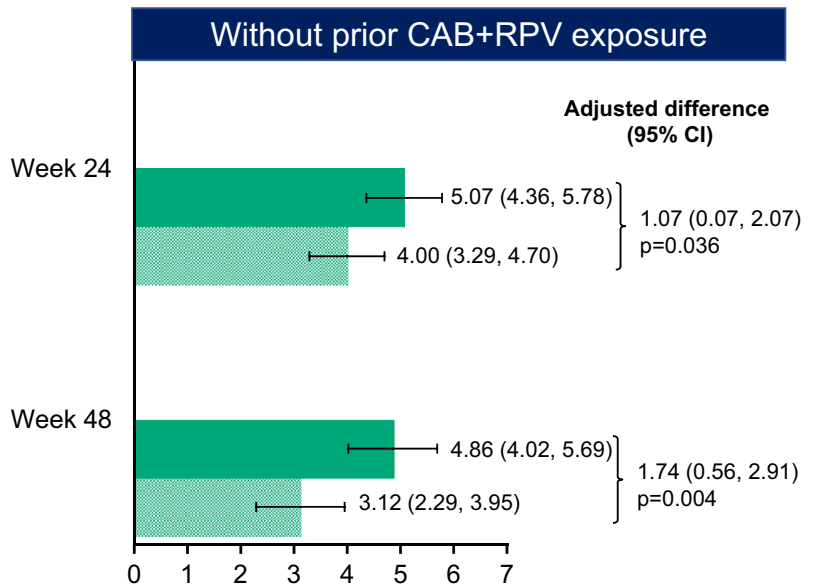

Week 24: Q8W, $n=192$; Q4W, $n=194$; Week 48: Q8W, $n=192$; $\mathrm{Q} 4 \mathrm{~W}, n=194$. Adjusted mean change from baseline calculated from an ANCOVA model including the following covariates: baseline score, sex at birth (female, male), age $(<50, \geq 50$ years), and race (white, non-white) for participants with no prior exposure; baseline score, sex at birth (female, male), age $(<50, \geq 50$ years), race (white, non-white), and prior exposure to CAB + RPV (1-24, > 24 weeks) for participants with prior exposure

\section{b}

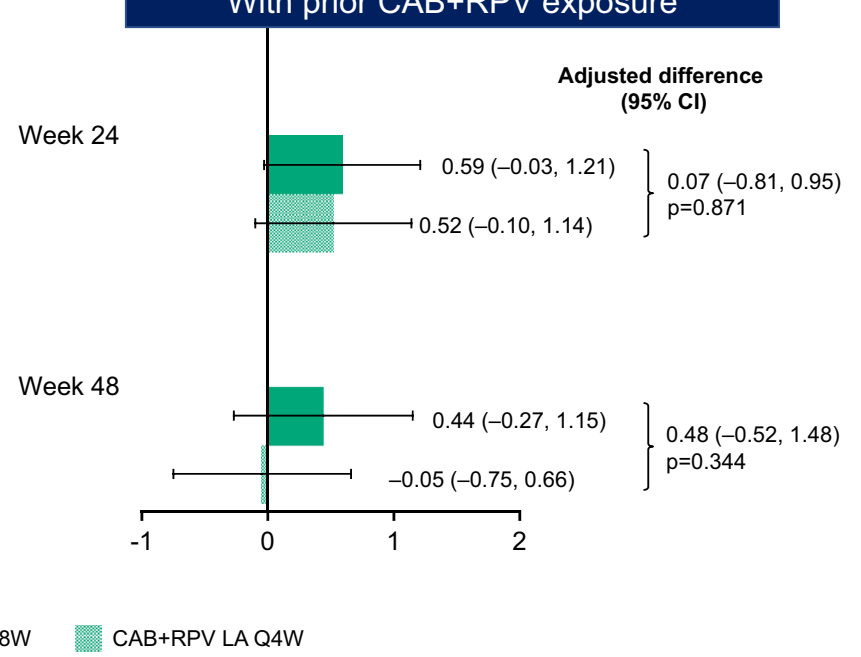

Fig. 4 Adjusted mean change from baseline in HIVTSQs by visit for participants without prior $\mathrm{CAB}+\mathrm{RPV}$ exposure (a) and with prior $\mathrm{CAB}+\mathrm{RPV}$ exposure $(\mathbf{b})^{\mathrm{a}}$. ANCOVA analysis of covariance, $C A B$ cabotegravir, CI confidence interval, HIVTSQs HIV Treatment Satisfaction Questionnaire (status version), $L A$ long-acting, $Q 4 W$ every 4 weeks, $Q 8 W$ every 8 weeks, $R P V$ rilpivirine. ${ }^{\text {a }}$ No prior exposure, Week 24: Q8W, $n=319$; Q4W, $n=323$; Week 48: Q8W, $n=319$; Q4W, $n=323$; prior exposure, Week 24: Q8W, $n=191$; Q4W, $n=193$; Week 48: Q8W, $n=191$; Q4W, $n=194$. Adjusted mean change from baseline calculated from an ANCOVA model including the following covariates: baseline score, sex at birth (female, male), age $(<50, \geq 50$ years), and race (white, non-white) for participants with no prior exposure; baseline score, sex at birth (female, male), age ( $<50, \geq 50$ years), race (white, non-white), and prior exposure to $\mathrm{CAB}+\mathrm{RPV}(1-24,>24$ weeks) for participants with prior exposure 
irrespective of prior $\mathrm{CAB}+\mathrm{RPV}$ exposure (adjusted mean change [95\% CI]: participants without prior exposure: Q8W 28.9/33.0 [27.9-29.9]; Q4W 27.1/33.0 [26.1-28.0]; participants with prior exposure: Q8W 28.9/33.0 [27.6-30.3]; Q4W 26.1/33.0 [24.7-27.4]). At Week 48, the scores representing the change in satisfaction relative to the prior treatment significantly favored $\mathrm{Q} 8 \mathrm{~W}$ over $\mathrm{Q} 4 \mathrm{~W}$, regardless of prior CAB + RPV exposure (adjusted difference [Q8W -Q4W] [95\% CI]: participants without prior exposure 1.9 [0.5-3.2], $p=0.008$; participants with prior exposure $2.9[1.0-4.8], p=0.004)$.

\subsection{Preference}

Among those participants in the $\mathrm{Q} 8 \mathrm{~W}$ arm with prior $\mathrm{CAB}+\mathrm{RPV}$ exposure responding to the preference question at Week 48, 94\% $(n=179 / 191)$ preferred CAB + RPV LA Q8W dosing versus Q4W (3\%,n=6/191) or daily oral dosing $(2 \%, n=4 / 191)$ (Fig. 5a). Participants without prior $\mathrm{CAB}+\mathrm{RPV}$ exposure who received CAB + RPV LA Q8W dosing with recorded responses to the preference question at Week 48 also preferred this regimen over daily oral dosing (98\%, $n=300 / 306)$ (Fig. 5a). Participants in the Q4W group (no experience of $\mathrm{Q} 8 \mathrm{~W}$ dosing) responding to the preference question at Week 48 preferred CAB + RPV LA Q4W dosing over daily oral dosing $(94 \%, n=468 / 497)$ (Fig. 5b). The most commonly cited reasons for long-acting preference were administration frequency and convenience, whereas the most common reasons supporting preference for daily oral dosing were the impact of side effects and convenience (electronic supplementary material Figure S4a, S4b, and $\mathrm{S} 4 \mathrm{c})$.
Fig. 5 Treatment preference at Week 48 in the Q8W arm (a) and Q4W arm (b). $C A B$ cabotegravir, $L A$ long-acting, $Q 4 W$ every 4 weeks, $Q 8 W$ every 8 weeks, $R P V$ rilpivirine, $S O C$ standard of care. a306 participants responded to the preference question. ${ }^{\mathrm{b}} 191$ participants responded to the preference question. ${ }^{\mathrm{c}} 497$ participants responded to the preference question. Figure 5a reprinted from The Lancet, Volume 396, Overton et al., Long-acting cabotegravir and rilpivirine dosed every 2 months in adults with HIV-1 infection (ATLAS-2M), 48-week results: a randomised, multicentre, open-label, phase 3b, non-inferiority study, Pages 1994-2005. Copyright (2020), with permission from Elsevier

a

\section{Participants in Q8W arm from SOC (no prior Q4W experience) $^{\mathrm{a}}$}

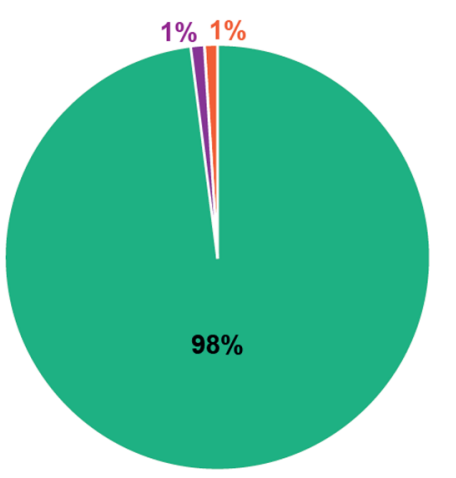

Q8W CAB+RPV LA

Daily oral
Participants in Q8W arm with prior Q4W experience in ATLAS ${ }^{b}$

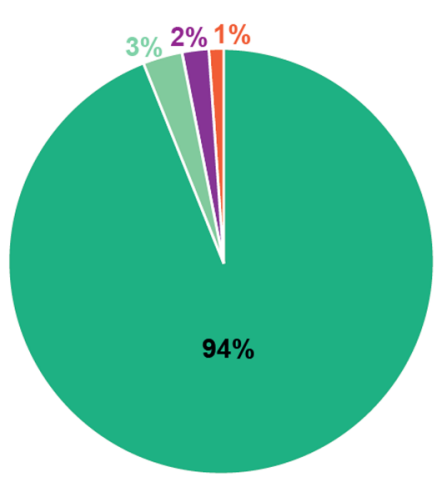

Q4W CAB+RPV LA

No preference

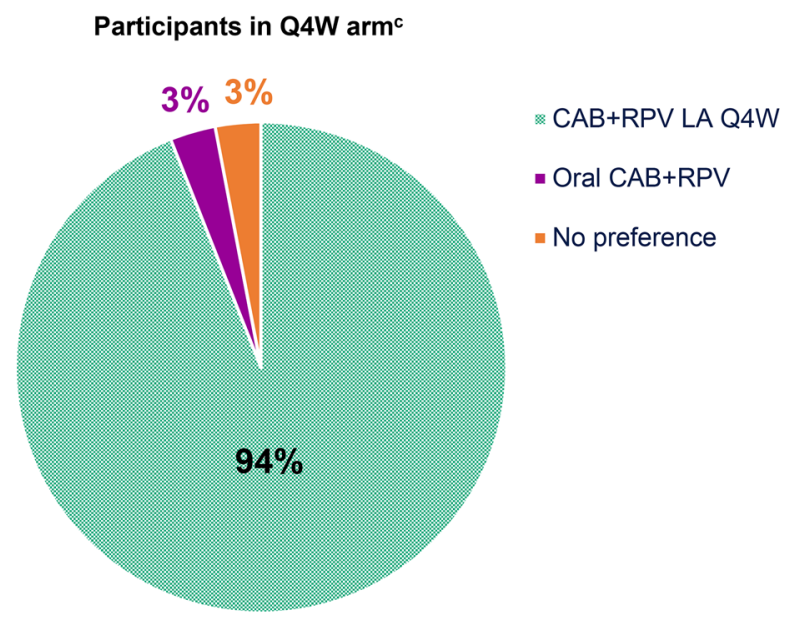




\subsection{Reason for Switch}

For participants originally randomized to the standard of care arm in ATLAS or coming from standard of care in the routine clinic, the reason most often selected for switching to long-acting therapy from standard of care was interest in new therapies $(85 \%, n=655 / 767)$ (electronic supplementary material Figure S2a). For participants originally randomized to the Q4W arm in ATLAS, the reason most often selected for continuing $\mathrm{CAB}+\mathrm{RPV}$ LA treatment was the convenience associated with injectable therapy $(87 \%, n=220 / 253)$ (electronic supplementary material Figure $\mathrm{S} 2 \mathrm{~b}$ ).

\section{Discussion}

The PRO data collected at Week 48 of the ATLAS-2M study demonstrate that both dosing regimens of CAB + RPV LA can improve and maintain treatment satisfaction and acceptance, supporting the therapeutic potential of the injectable regimen and highlighting participants' preference for longacting therapy over daily oral dosing. These results complement the high overall rates of virologic suppression and favorable safety and tolerability profiles observed for $\mathrm{CAB}+\mathrm{RPV}$ LA Q8W and Q4W in ATLAS-2M [18]. The $\mathrm{PRO}$ analysis was stratified by prior $\mathrm{CAB}+\mathrm{RPV}$ exposure to accommodate the difference in treatments assessed at baseline. As such, participants without prior CAB + RPV exposure were reporting their experience with daily oral therapy at baseline, whereas participants with prior $\mathrm{CAB}+\mathrm{RPV}$ exposure were reporting their experience with the $\mathrm{Q} 4 \mathrm{~W}$ long-acting regimen they received during the ATLAS study.

Although ISRs were frequent during ATLAS-2M [18], particularly pain events, they seldom led to withdrawal and nearly all participants preferred the long-acting regimen to their previous oral therapy. The majority of participants reported high acceptability of pain and local reactions at early timepoints in the study, with acceptability increasing with subsequent injections. These improvements are mirrored by the reduction in frequency of ISRs over time observed in the safety analysis of ATLAS-2M [18], providing evidence that ISRs associated with $C A B+R P V$ LA become perceived as less burdensome over time by participants. Despite ISRs being more prevalent per visit with the Q8W regimen versus the Q4W regimen, although offset over time by less frequent dosing, similar improvements in acceptability of pain and local reactions were observed regardless of dosing regimen (Q8W vs. Q4W). Therefore, it could be argued that the increased volume of liquid delivered with the Q8W regimen does not impact the tolerability or acceptability of injections (Q8W $2 \times 3 \mathrm{~mL}$; Q4W $2 \times 2 \mathrm{~mL}$ ).

With respect to treatment satisfaction and acceptance, participants entering ATLAS-2M with only prior experience of daily oral therapy reported large improvements at Weeks 24 and 48 across both long-acting groups. The magnitude of the improvements were consistent with the improvements in satisfaction and acceptance observed in participants in the ATLAS and FLAIR phase 3 clinical studies, which for ATLAS was proven to meet the minimal clinically important difference threshold [16, 17, 22]. For participants entering ATLAS-2M having received CAB + RPV LA Q4W in ATLAS, treatment satisfaction and acceptance was high at baseline and maintained through Week 48 in both long-acting groups. Further, most participants transitioning from ATLAS switched from the $\mathrm{Q} 4 \mathrm{~W}$ regimen and had therefore accumulated $\geq 96$ weeks of CAB + RPV LA exposure by Week 48 of ATLAS-2M $(65 \%, n=253 / 391)$. These longer-term findings are consistent with the high participant satisfaction reported at Week 96 in FLAIR, and when taken together suggest that the benefits of longacting therapy can be sustained over $\sim 2$ years [38]. Over time, minor fluctuations are to be expected in treatment satisfaction and acceptance scores, both of which remained at high levels throughout the study. For participants with prior $\mathrm{CAB}+\mathrm{RPV}$ exposure, the negative trend in treatment acceptance score was more pronounced for the Q4W arm. This could be attributable to some participants' disappointment with receiving the same regimen they had received in ATLAS, missing the opportunity to initiate the less frequent, potentially more desirable, Q8W dosing regimen. For treatment satisfaction scores, small decreases were observed between Weeks 24 and 48, irrespective of participants' prior $\mathrm{CAB}+\mathrm{RPV}$ exposure. For participants without prior $\mathrm{CAB}+\mathrm{RPV}$ exposure and participants randomized to the $\mathrm{Q} 8 \mathrm{~W}$ regimen, this may be a consequence of inflated satisfaction scores recorded at Week 24 due to the initial excitement associated with receiving a novel therapeutic or being randomized to the less frequent dosing regimen.

For participants with prior $\mathrm{CAB}+\mathrm{RPV}$ exposure, high values for both treatment acceptance and satisfaction (HIVTSQs) were maintained across treatment regimens; however, no significant differences were observed between Q8W and Q4W dosing. A significant difference favoring the Q8W regimen was only observed in treatment satisfaction (HIVTSQs) for participants without prior CAB + RPV exposure. This can be partially attributed to the ceiling effects observed with the use of the ACCEPT and HIVTSQs instruments, which mean the high baseline values for participants with prior $\mathrm{CAB}+\mathrm{RPV}$ exposure do not permit the full magnitude of treatment effects to become apparent. With the use of HIVTSQc, developed to account for ceiling effects, the change in satisfaction relative to prior treatment scores significantly favored Q8W over Q4W at Week 48, irrespective of prior exposure.

Preference questionnaires revealed that almost all participants with experience of both the Q8W and Q4W regimens 
preferred Q8W dosing, the reasons for which highlight the perceived value of reduced dosing frequencies and greater convenience for participants. In addition to the participantreported reasons for preference, the $\mathrm{Q} 8 \mathrm{~W}$ regimen has been observed to have comparable efficacy and tolerability to the Q4W regimen [18]. Further, the Q8W regimen offers fewer opportunities for missed injections and requires half the number of clinical visits per year. Whilst previous studies have reported strong preferences for the long-acting regimen over daily oral antiretroviral therapy $[16,17,20$, 22], ATLAS-2M represents the first phase 3 clinical study to report participant preference for $\mathrm{Q} 8 \mathrm{~W}$ dosing over $\mathrm{Q} 4 \mathrm{~W}$ dosing, consistent with the greater increases in satisfaction reported in Q8W arm participants compared with those in the $\mathrm{Q} 4 \mathrm{~W}$ arm. The Q8W regimen has several potential realworld advantages over Q4W dosing, as it facilitates fewer clinical interventions as well as reducing the total number of treatments/visits to six per year (excluding first loading dose).

Overall, the findings of these analyses provide robust evidence supporting the benefits of long-acting therapy outside of the clinically focused parameters of efficacy and safety, prioritizing an evaluation of the patient's experience and preferences. This sentiment is in keeping with the general trend towards a more patient-centered focus in the care of people living with HIV-1. Patient satisfaction may contribute to individual improvements in adherence, which in turn translates to better management of HIV [23]. Increasing patient satisfaction is also vital to improving overall quality of life for people living with HIV-1, a metric of ever-increasing importance as the efficacy, tolerability, and accessibility of HIV therapeutics continue to improve [1]. The results collected at Week 48 of the ATLAS-2M study are illustrative of the therapeutic potential of CAB + RPV LA, not only as an efficacious and well-tolerated maintenance regimen, but also as a regimen with the potential to facilitate improvements in adherence and a person's overall quality of life. The translation of long-acting therapies into everyday widespread clinical practice warrants comprehensive investigation and is undergoing evaluation [39].

\subsection{Limitations}

The present study has several limitations. Although ATLAS$2 \mathrm{M}$ met its enrollment target of $25 \%$ female participants (sex at birth) and also included $>25 \%$ of participants aged $\geq 50$ years, the enrolled population did not include large proportions of other under-represented groups, including people from racial and ethnic minority groups, transgender individuals, and individuals with historic suboptimal adherence [18]. In addition, the enrolled population may reflect a select population of people living with HIV-1 interested in long-acting therapies. Further research, including real-world evidence, is ongoing or planned to evaluate $\mathrm{CAB}+\mathrm{RPV}$ LA in different patient demographics and describe use in broader populations of people living with HIV-1. For example, the ongoing LATITUDE study (NCT03635788) is investigating $\mathrm{CAB}+\mathrm{RPV}$ LA in a population with historic suboptimal adherence [40]. In addition, studies that directly compare $\mathrm{CAB}+\mathrm{RPV}$ LA Q8W with contemporary standard of care antiretroviral regimens are warranted and have been planned, such as the upcoming SOLAR (NCT04542070) study, which will evaluate $\mathrm{CAB}+\mathrm{RPV}$ LA dosed every 2 months compared with a bictegravir/emtricitabine/tenofovir alafenamide-based oral daily regimen [41]. A small proportion of study participants $(14 \% ; n=147 / 1020$ [respondents]) answered "cost of alternative options/current HIV drug or to continue receiving free study drug" as their reason for switching to/continuing long-acting therapy. This result could have inadvertently revealed a source of unconscious bias, which may have augmented some PRO item scores. The absence of a therapeutic alternative for participants due to economic constraints may also qualify as undue influence. However, participants provided written informed consent, which was approved by national, regional, or investigational center ethics committees or institutional review board, in accordance with The International Conference on Harmonisation Good Clinical Practice Guideline and applicable country-specific requirements.

\section{Conclusions}

Irrespective of dosing regimen, $\mathrm{CAB}+\mathrm{RPV}$ LA was associated with large increases in treatment satisfaction and high acceptance of ISRs for those naive to CAB + RPV. For those with prior experience of the intramuscular regimen, $\mathrm{CAB}+\mathrm{RPV}$ LA maintained high levels of satisfaction and acceptance. Most participants preferred Q8W and Q4W dosing over daily oral dosing, with Q8W also preferred over Q4W dosing. The PRO data presented here, along with the high rates of virologic suppression, low rates of virologic failure, and favorable safety and tolerability profiles presented in the primary analysis, support the therapeutic potential of monthly or every 2 months CAB + RPV LA and highlight participants' preference for long-acting therapy over daily oral dosing.

Supplementary Information The online version contains supplementary material available at https://doi.org/10.1007/s40271-021-00524-0.

Acknowledgements We thank everyone who has contributed to the success of the study: all study participants and their families, and the clinical investigators and their staff. Professional medical writing and editorial assistance was provided by Euan Paul at SciMentum (Nucleus Global) and funded by ViiV Healthcare. 


\section{Declarations}

Funding ATLAS-2M is funded by ViiV Healthcare and Janssen. Openaccess was funded by ViiV Healthcare.

Conflict of interest VC, PDB, KYS, and WRS are employees of ViiV Healthcare and stockholders of GlaxoSmithKline. ETO has received research support to his institution during the conduct of this study and served as a consultant for Gilead, Merck, Thera Technologies, and ViiV Healthcare, outside of the submitted work. AM has received research funding and consulting fees from ViiV Healthcare, Gilead, Janssen, and Merck as well as consulting fees from Shionogi, all outside the submitted work. SS reports grants from ViiV Healthcare during the conduct of the study. SV and RVS are employees and stockholders of Janssen, Pharmaceutical Companies of Johnson \& Johnson. YW is an employee and stockholder of GlaxoSmithKline. KJH was an employee of ViiV Healthcare and stockholder of GlaxoSmithKline during the conduct of the study and is now an employee of Horizon Therapeutics. MSS was an employee of ViiV Healthcare and stockholder of GlaxoSmithKline during the conduct of the study and is now an employee of Hengrui USA. DAM was an employee of ViiV Healthcare and stockholder of GlaxoSmithKline during the conduct of the study and is now an employee of Brii Biosciences.

Ethics approval ATLAS-2M was conducted in accordance with the principles founded in the Declaration of Helsinki and with Good Clinical Practice. The study protocol was approved by an institutional review board or ethics committee.

Consent to participate All participants provided written informed consent.

Consent for publication All participants provided written informed consent.

Availability of data and material Data sharing requests will be considered upon written request to the corresponding author. Deidentified participant data or other prespecified data will be available subject to a written proposal and a signed data sharing agreement.

Code availability The datasets used and/or analyzed during the current study are available from the corresponding author on reasonable request.

Authors' contributions ETO, AM, and SS participated in the collection and interpretation of the data. VC, PDB, SV, RVS, YW, KJH, MSS, DAM, KYS, and WRS were responsible for the development, conception, and data analysis of the study. All authors contributed to the review and development of the manuscript.

Open Access This article is licensed under a Creative Commons Attribution-NonCommercial 4.0 International License, which permits any non-commercial use, sharing, adaptation, distribution and reproduction in any medium or format, as long as you give appropriate credit to the original author(s) and the source, provide a link to the Creative Commons licence, and indicate if changes were made. The images or other third party material in this article are included in the article's Creative Commons licence, unless indicated otherwise in a credit line to the material. If material is not included in the article's Creative Commons licence and your intended use is not permitted by statutory regulation or exceeds the permitted use, you will need to obtain permission directly from the copyright holder. To view a copy of this licence, visit http://creativecommons.org/licenses/by-nc/4.0/.

\section{References}

1. Lee FJ, Amin J, Carr A. Efficacy of initial antiretroviral therapy for HIV-1 infection in adults: a systematic review and meta-analysis of 114 studies with up to 144 weeks' follow-up. PLoSOne. 2014;9:e97482.

2. Vitoria M, Ford N, Doherty M, Flexner C. Simplification of antiretroviral therapy: a necessary step in the public health response to HIV/AIDS in resource-limited settings. Antivir Ther. 2014;19(Suppl 3):31-7.

3. Clay PG, Nag S, Graham CM, Narayanan S. Meta-analysis of studies comparing single and multi-tablet fixed dose combination HIV treatment regimens. Medicine. 2015;94:e1677.

4. Shubber Z, Mills EJ, Nachega JB, Vreeman R, Freitas M, Bock $\mathrm{P}$, et al. Patient-reported barriers to adherence to antiretroviral therapy: a systematic review and meta-analysis. PLoS Med. 2016;13:e1002183.

5. De Los Rios P, Young B, Marcotullio S, Punekar Y, Koteff J, Ustianowski A, et al. 1329. Experiences and emotional challenges of antiretroviral treatment (ART) - findings from the Positive Perspectives Study. Open Forum Infect Dis. 2019;6(Suppl 2):S481.

6. Swindells S, Flexner C, Fletcher CV, Jacobson JM. The critical need for alternative antiretroviral formulations, and obstacles to their development. J Infect Dis. 2011;204:669-74.

7. Nachega JB, Marconi VC, van Zyl GU, Gardner EM, Preiser W, Hong SY, et al. HIV treatment adherence, drug resistance, virologic failure: evolving concepts. Infect Disord Drug Targets. 2011;11:167-74.

8. Li JZ, Paredes R, Ribaudo HJ, Svarovskaia ES, Metzner KJ, Kozal MJ, et al. Low-frequency HIV-1 drug resistance mutations and risk of NNRTI-based antiretroviral treatment failure: a systematic review and pooled analysis. J Am Med Assoc. 2011;305:1327-35.

9. Nega J, Taye S, Million Y, Rodrigo C, Eshetie S. Antiretroviral treatment failure and associated factors among HIV patients on first-line antiretroviral treatment in Sekota, northeast Ethiopia. AIDS Res Ther. 2020;17:39.

10. Ahmed M, Merga H, Jarso H. Predictors of virological treatment failure among adult HIV patients on first-line antiretroviral therapy in Woldia and Dessie hospitals, Northeast Ethiopia: a case-control study. BMC Infect Dis. 2019;19:305.

11. Williams J, Sayles HR, Meza JL, Sayre P, Sandkovsky U, Gendelman HE, et al. Long-acting parenteral nanoformulated antiretroviral therapy: interest and attitudes of HIV-infected patients. Nanomedicine (Lond). 2013;8:1807-13.

12. Dandachi D, Dang BN, Lucari B, Swindells S, Giordano TP. Acceptability and preferences for long-acting antiretroviral formulations among people with HIV infection. AIDS Care. 2020: https://doi.org/10.1080/09540121.2020.1764906 [online ahead of print].

13. Weld ED, Rana MS, Dallas RH, Camacho-Gonzalez AF, Ryscavage $\mathrm{P}$, Gaur $\mathrm{AH}$, et al. Interest of youth living with hiv in long-acting antiretrovirals. J Acquir Immune Defic Syndr. 2019;80:190-7.

14. Philbin MM, Parish C, Kinnard EN, Reed SE, Kerrigan D, Alcaide M, et al. A multi-site study of women living with HIV's perceived barriers to, and interest in, long-acting injectable anti-retroviral therapy. J Acquir Immune Defic Syndr. 2020;8:263-70.

15. Kerrigan D, Sanchez Karver T, Muraleetharan O, Savage V, Mbwambo J, Donastorg Y, et al. "A dream come true": perspectives on long-acting injectable antiretroviral therapy among female sex workers living with HIV from the Dominican Republic and Tanzania. PLoSOne. 2020;15:

16. Orkin C, Arasteh K, Górgolas Hernández-Mora M, Pokrovsky V, Overton ET, Girard PM, et al. Long-acting cabotegravir and rilpivirine after oral induction for HIV-1 infection. N Engl J Med. 2020;382:1124-35. 
17. Swindells S, Andrade-Villanueva JF, Richmond GJ, Rizzardini G, Baumgarten A, Masiá M, et al. Long-acting cabotegravir and rilpivirine for maintenance of HIV-1 suppression. N Engl J Med. 2020;382:1112-23.

18. Overton ET, Richmond G, Rizzardini G, Jaeger H, Orrell C, Nagimova F, et al. Long-acting cabotegravir and rilpivirine dosed every 2 months in adults with HIV-1 infection (ATLAS-2M), 48-week results: a randomised, multicentre, open-label, phase 3b, non-inferiority study. Lancet. 2021;396:1994-2005.

19. Margolis DA, Gonzalez-Garcia J, Stellbrink HJ, Eron JJ, Yazdanpanah Y, Podzamczer D, et al. Long-acting intramuscular cabotegravir and rilpivirine in adults with HIV-1 infection (LATTE-2): 96-week results of a randomised, open-label, phase $2 b$, non-inferiority trial. Lancet. 2017;390:1499-510.

20. Kerrigan D, Mantsios A, Gorgolas M, Montes ML, Pulido F, Brinson C, et al. Experiences with long acting injectable ART: a qualitative study among PLHIV participating in a Phase II study of cabotegravir + rilpivirine (LATTE-2) in the United States and Spain. PLoSOne. 2018;13:e0190487.

21. Rizzardini G, Overton ET, Orkin C, Swindells S, Arasteh K, Górgolas Hernández-Mora M, et al. Long-acting injectable cabotegravir + rilpivirine for HIV maintenance therapy: week 48 pooled analysis of phase 3 ATLAS and FLAIR trials. J Acquir Immune Defic Syndr. 2020;85:498-506.

22. Murray M, Antela A, Mills A, Huang J, Jäger H, Bernal E, et al. Patient-reported outcomes in ATLAS and FLAIR participants on long-acting regimens of cabotegravir and rilpivirine over 48 weeks. AIDS Behav. 2020;24:3533-44.

23. Dang BN, Westbrook RA, Black WC, Rodriguez-Barradas MC, Giordano TP. Examining the link between patient satisfaction and adherence to HIV care: a structural equation model. PLoSOne. 2013;8:e54729.

24. Bulage L, Ssewanyana I, Nankabirwa V, Nsubuga F, Kihembo C, Pande G, et al. Factors associated with virological non-suppression among HIV-positive patients on antiretroviral therapy in Uganda, August 2014-July 2015. BMC Infect Dis. 2017;17:326.

25. Biset Ayalew M. Mortality and its predictors among HIV infected patients taking antiretroviral treatment in Ethiopia: a systematic review. AIDS Res Treat. 2017;2017:5415298.

26. Rai S, Mahapatra B, Sircar S, Raj PY, Venkatesh S, Shaukat M, et al. Adherence to antiretroviral therapy and its effect on survival of HIV-infected individuals in Jharkhand, India. PLoSOne. 2013;8:e66860.

27. Llibre JM, Hung CC, Brinson C, Castelli F, Girard PM, Kahl LP, et al. Efficacy, safety, and tolerability of dolutegravir-rilpivirine for the maintenance of virological suppression in adults with HIV1: phase 3, randomised, non-inferiority SWORD-1 and SWORD-2 studies. Lancet. 2018;391:839-49.

28. van Wyk J, Ajana F, Bisshop F, De Wit S, Osiyemi O, Portilla Sogorb J, et al. Efficacy and safety of switching to dolutegravir/ lamivudine fixed-dose 2-drug regimen vs continuing a tenofovir alafenamide-based 3- or 4-drug regimen for maintenance of virologic suppression in adults living with human immunodeficiency virus type 1 : phase 3 , randomized, noninferiority TANGO study. Clin Infect Dis. 2020;71:1920-9.
29. Cahn P, Madero JS, Arribas JR, Antinori A, Ortiz R, Clarke AE, et al. Dolutegravir plus lamivudine versus dolutegravir plus tenofovir disoproxil fumarate and emtricitabine in antiretroviral-naive adults with HIV-1 infection (GEMINI-1 and GEMINI-2): week 48 results from two multicentre, double-blind, randomised, noninferiority, phase 3 trials. Lancet. 2019;393:143-55.

30. World Medical Association. World Medical Association Declaration of Helsinki: ethical principles for medical research involving human subjects. J Am Med Assoc. 2013;310:2191-4.

31. Dixon JR, Jr. The international conference on harmonization good clinical practice guideline. Qual Assur. 1998;6:65-74.

32. Chevat C, Viala-Danten M, Dias-Barbosa C, Nguyen VH. Development and psychometric validation of a self-administered questionnaire assessing the acceptance of influenza vaccination: the Vaccinees' Perception of Injection (VAPI) questionnaire. Health Qual Life Outcomes. 2009;7:21.

33. Arnould B, Gilet H, Patrick DL, Acquadro C. Item reduction, scoring, and first validation of the ACCEPTance by the Patients of their Treatment $\left(\right.$ ACCEPT $^{\odot}$ ) Questionnaire. Patient. 2017;10:81-92.

34. Marant C, Longin J, Gauchoux R, Arnould B, Spizak C, Marrel A, et al. Long-term treatment acceptance: what is it, and how can it be assessed? Patient. 2012;5:239-49.

35. Woodcock A, Bradley C. Validation of the revised 10-item HIV Treatment Satisfaction Questionnaire status version and new change version. Value Health. 2006;9:320-33.

36. Bradley C, Plowright R, Stewart J, Valentine J, Witthaus E. The Diabetes Treatment Satisfaction Questionnaire change version (DTSQc) evaluated in insulin glargine trials shows greater responsiveness to improvements than the original DTSQ. Health Qual Life Outcomes. 2007;5:57.

37. Romaine J, Murray M, Bradley C. PIH62 investigating the responsiveness to change of the hiv treatment satisfaction questionnaire change version (HIVTSQC) in overcoming ceiling effects in the hiv treatment satisfaction questionnaire status version (HIVTSQS). Value Health. 2019;22:S638-9.

38. Orkin C, Oka S, Philibert P, Brinson C, Bassa A, Gusev D, et al. Long-acting cabotegravir plus rilpivirine for treatment in adults with HIV-1 infection: 96-week results of the randomised, openlabel, phase 3 FLAIR study. Lancet HIV. 2021;8:e185-96.

39. ClinicalTrials.gov. Study to identify and determine best implementation practices for injectable cabotegravir + rilpivirine in the United States (NCT04001803). https://www.clinicaltrials.gov/ct2/ show/NCT04001803. Accessed 22 Oct 2019.

40. ClinicalTrials.gov. The LATITUDE study: long-acting therapy to improve treatment success in daily life (NCT03635788). https:// www.clinicaltrials.gov/ct2/show/NCT03635788. Accessed 18 Mar 2020.

41. International Conference on Harmonisation Good Clinical Practice Network. A Study to evaluate efficacy and safety of cabotegravir (CAB) long acting (LA) Plus (+) Rilpivirine (RPV) LA Versus BIKTARVY ${ }^{\circledR}(\mathrm{BIK})$ in participants with human immunodeficiency virus (HIV)-1 who are virologically suppressed. https:// ichgcp.net/clinical-trials-registry/NCT04542070. Accessed 9 Oct 2020. 


\section{Authors and Affiliations}

Vasiliki Chounta ${ }^{1}$ (D) Edgar T. Overton ${ }^{2} \cdot$ Anthony Mills $^{3} \cdot$ Susan Swindells ${ }^{4} \cdot$ Paul D. Benn $^{1} \cdot$ Simon Vanveggel $^{5}$. Rodica van Solingen-Ristea ${ }^{5}$ Y Yuanyuan Wang ${ }^{6} \cdot$ Krischan J. Hudson $^{7,8} \cdot$ Mark S. Shaefer $^{7,9} \cdot$ David A. Margolis ${ }^{7,10}$. Kimberly Y. Smith ${ }^{7}$. William R. Spreen ${ }^{7}$

Vasiliki Chounta

vasiliki.x.chounta@viivhealthcare.com

1 ViiV Healthcare, Brentford, UK

2 University of Alabama at Birmingham, Birmingham, AL, USA

3 Men's Health Foundation, Los Angeles, CA, USA

4 University of Nebraska Medical Center, Omaha, NE, USA

5 Janssen Pharmaceutica NV, Beerse, Belgium
GlaxoSmithKline, Collegeville, PA, USA

7 ViiV Healthcare, Research Triangle Park, NC, USA

8 Horizon Therapeutics, Lake Forest, IL, USA

9 Hengrui USA, East Windsor, NJ, USA

10 Brii Biosciences, Durham, NC, USA 